\title{
Statistical distribution, host for encrypted information
}

\author{
L. Rebollo-Neira \\ Aston University \\ Birmingham B4 7ET, United Kingdom A. Plastino \\ Instituto de Física La Plata (IFLP) \\ Universidad Nacional de La Plata and CONICET * \\ C.C. 727,1900 La Plata, Argentina
}

\begin{abstract}
The statistical distribution, when determined from an incomplete set of constraints, is shown to be suitable as host for encrypted information. We design an encoding/decoding scheme to embed such a distribution with hidden information. The encryption security is based on the extreme instability of the encoding procedure. The essential feature of the proposed system lies in the fact that the key for retrieving the code is generated by random perturbations of very small value. The security of the proposed encryption relies on the security to interchange the secret key. Hence, it appears as a good complement to the quantum key distribution protocol.
\end{abstract}

PACS: 05.20.-y, 02.50.Tt, 02.30.Zz, 07.05.Kf

\section{Introduction}

Cryptography is the art of code making and cryptology the art of secure communications. Recently, quantum mechanics has made a remarkable entry in the field [1-3]. The most straightforward application of quantum cryptology is the distribution of secret keys. This problem is refereed in the cryptography literature to as the key distribution problem. Classical methods for securing a secret key are based on the assumed difficulty of computing certain functions $[4,5]$. Quantum encryption provides a way of agreeing on a secret key without making this assumption. The first

${ }^{*}$ Argentina's National Research Council 
quantum key distribution protocol was proposed in 1984 by Bennett and Brassard [6] and there are already rigorous proofs of its security [7-9]. The list of recent contributions concerning the security and implementation of quantum key distribution protocols is certainly extensive. Just as a sample one should mention [10-14].

The amount of information that can be transmitted by a quantum transmission is not very large, but by means of secret-key cryptographic algorithms a large amount of information can be secured. In this paper we set the foundations for a statistical distribution based encryption procedures, which will be shown to be good complements to quantum cryptology. It will be here demonstrated that the statistical distribution of a physical system is a suitable host for encrypted information and we will discuss a method for embedding encrypted messages without affecting the physical content of the distribution.

The fact that a physical system can be assumed to be well described by a particular parametric class of statistical distribution entails, in most situations, the assumption of a great deal of prior information. Indeed, the functional form of most celebrated statistical distribution (Gibbs-Boltzmann, Fermi-Dirac, Bose-Einstein distributions, etc) can be derived from a few constraints expressed as mean values of some observable, and the optimisation of a convex function called entropy or information measure [15]. Thus, if a so obtained distribution happens to be the right one to describe a particular system, one can think of the optimisation process as replacing the information required to determine a unique distribution for the system.

We would like to think that, when one "guesses" (or derives) a distribution from incomplete information, one also generates an "invisible reservoir" to place information. This is not an original remark, of course, but an elemental result of linear algebra: associated to a rank deficient transformation there are two spaces, the range and null spaces of the transformation. The latter is an "invisible" space in the sense that all its elements are mapped to zero by the transformation. 
In this Communication we show that the invisible reservoir is an appropriate host for storing covert information. We present an encoding/decoding scheme that allows to store a great amount of hidden information as storing the distribution of a physical system. The main idea is to make use of the null space of the transformation generated by the constraints that should be fulfilled by the distribution. The security of the system is guaranteed by designing a (in the popular meaning of the vocable, not in the technical one) "chaotic" encoding procedure. This is achieved by means of random perturbations to an extremely sensitive encoding process. The random perturbations of very small values provide one thereby with the key for recovering the code. This is the most remarkable feature of the our proposal: the key for retrieving the hidden information is just a tiny number that accounts for the perturbation that has been used for encoding purposes. Hence, the relevance of this proposal in relation to quantum cryptology, and vice versa, since the security of our proposal depends on a secure key interchange. It is appropriate to remark that the most notable difference between this approach and chaotic cryptosystems $[16,17]$ is that the theory underlying our approach is essentially a linear one.

The idea of making use of an "unstable system" for encryption has been successfully applied to over-sampling of Fourier coefficients for transmitting hidden messages as transmitting a signal [18]. Here we use equivalent ideas. We assume that, in addition to some constraints, we have the information on the process by which the statistical distribution is univocally determined. This process is usually the optimisation of a convex function (entropy). The particular expression for the entropy may be a matter of controversy, though. For our purposes the choice of the entropic measure is not relevant at all. What is important here is the convexity property to ensure a unique solution. The selection of the appropriate entropic measure is crucial, of course, to determine the right distribution for the physical system. Nevertheless, this has no relation whatsoever with our encryption scheme. 
The paper is organised as follows: In section II we introduce the notation together with an encoding/decoding scheme for embedding a statistical distribution with hidden information. The procedure is illustrated by a numerical simulation in section III and some conclusions are drawn in section IV.

\section{Embedding the statistic distribution}

We restrict considerations to finite dimensional classical statistic systems, or equivalently, to a quantum system represented by a distribution constructed from commutative operators. In both cases the mean value of, say $M$, physical observations $x_{1}^{o}, x_{2}^{o}, \ldots, x_{i}^{o}, \ldots x_{M}^{o}$, each of which is the expectation value of a random variable that takes values $x_{i, n} ; n=1, \ldots, N$ according to a probability distribution $p_{n} ; n=1, \ldots, N$ is expressed as:

$$
\begin{aligned}
x_{i}^{o} & =\sum_{n=1}^{N} p_{n} x_{i, n} \quad ; \quad i=1, \ldots, M \\
1 & =\sum_{n=1}^{N} p_{n}
\end{aligned}
$$

Usually the number $M$ of available measurements is much less than the dimension $N$ of the probability space. In order to assert a unique distribution for the system at hand one has to adopt a decision criterion, which is frequently implemented through the maximisation of a convex measure on the probability distribution. Such a measure, called entropy or information measure, takes different forms. Here we simple assume that the distribution characterising a given physical system is agreed to be determined by a fixed set of constraints of the form (11) and the optimisation of a convex function that we denote $S$.

For the sake of a handy notation we use Dirac notation to represent vectors. Thus, the probability distribution is represented as the ket $|p\rangle \in \mathbb{R}^{N}$ which, by 
denoting as $|n\rangle, n=1, \ldots, N$ the standard basis in $\mathbb{R}^{N}$, can be expressed as

$$
|p\rangle=\sum_{n=1}^{N}|n\rangle\langle n \mid p\rangle=\sum_{n=1}^{N} p_{n}|n\rangle .
$$

We also define a vector $\left|x^{o}\right\rangle \in \mathbb{R}^{M}$ of components $x_{1}^{o}, x_{2}^{o}, \ldots, x_{M}^{o}, 1$ and an operator $\hat{A}: \mathbb{R}^{N} \rightarrow \mathbb{R}^{M+1}$ given by

$$
\hat{A}=\sum_{n=1}^{N}\left|x_{n}\right\rangle\langle n| .
$$

Vectors $\left|x_{n}\right\rangle \in \mathbb{R}^{M+1}, n=1, \ldots, N$ are defined in such a way that $\left\langle i \mid x_{n}\right\rangle=x_{i, n}, i=$ $1, \ldots, M+1$ with $x_{M+1, n}=1$. Hence,

$$
\left|x_{n}\right\rangle=\sum_{i=1}^{M+1}|i\rangle\left\langle i \mid x_{n}\right\rangle=\sum_{i=1}^{M+1} x_{i, n}|i\rangle .
$$

We are now in a position to joint the constraints (11) together in the equation

$$
\left|x^{o}\right\rangle=\hat{A}|p\rangle
$$

Since $\hat{A}$ is a rank deficient operator, we know from elemental linear algebra that the general solution to the under-determined system (5) can be expressed in the form:

$$
|p\rangle=\hat{A}^{\prime-1}\left|x^{o}\right\rangle+\left|p^{\prime}\right\rangle,
$$

where $\hat{A}^{\prime-1}$ is the pseudo inverse of $\hat{A}$ (i.e. the inverse of the restriction of $\hat{A}$ to range $(\hat{A}))$ and $\left|p^{\prime}\right\rangle$ a vector in the null space of that operator. Consequently, all the information the probability distribution contains concerning the data is expressible in the fashion $\hat{A}^{\prime}\left|x^{o}\right\rangle$. On the contrary, the component $\left|p^{\prime}\right\rangle$ is completely independent of the data, but strongly dependent on the selection criterion that is adopted to decide on one particular solution among the infinitely many solutions the system (6) has.

The fact that all distributions of the form $|\tilde{p}\rangle=\hat{A}^{\prime-1}\left|x^{o}\right\rangle+\left|p^{\prime}\right\rangle$ with $\left|p^{\prime}\right\rangle \in$ $\operatorname{null}(\hat{A})$ are capable of reproducing the constraints vector $\left|x^{o}\right\rangle$ provides us with a 
framework for the purpose of storing encrypted information while storing the statistical distribution of a physical system. At the encoding step we make the following assumptions:

i) The number $M+1$ of the independent linear equations which are used to determine the statistical distribution of a given system is fixed. The expected values generating the equations are assumed to be known.

ii) The probability distribution characterising the system arises by optimisation of a convex function $S$, subjected to the $M+1$ linear constraints described above.

Assumptions i) and ii) entail the availability, at this stage, of vector $|p\rangle$ and operator $\hat{A}$. The vectors spanning the range and null spaces of this operator can be determined by computing the the eigenvectors of operator $\hat{G}=\hat{A}^{\dagger} \hat{A}$. Let us denote as $\left|\eta_{n}\right\rangle, n=1, \ldots, N-(M+1)$ the normalised eigenvectors corresponding to zero eigenvalues. We use these vectors to define operator $\hat{U}: \mathbb{R}^{N} \mapsto \mathbb{R}^{N-(M+1)}$ as

$$
\hat{U}=\sum_{n=1}^{N-M-1}|n\rangle\left\langle\eta_{n}\right|
$$

This operator is termed decoding operator, and its adjoint, $\hat{U}^{\dagger}$, encoding operator. Using $\hat{U}^{\dagger}$ a basic code of $N-(M+1)$ numbers is constructed as follows: Let the $N-(M+1)$ numbers be the $N-(M+1)$-components $\langle n \mid q\rangle=q_{n}, n=1, \ldots, N-$ $(M+1)$ of vector $|q\rangle \in \mathbb{R}^{N-(M+1)}$ and define:

$$
\left|p_{c}\right\rangle=\hat{U}^{\dagger}|q\rangle=\sum_{n=1}^{N-M-1}\left|\eta_{n}\right\rangle\langle n \mid q\rangle
$$

Given a distribution $|p\rangle$, amenable to be determined from the optimisation of an entropy measure $S$ and a set of $M+1$ constraints, the code $|q\rangle$ is embedded in the distribution through the process below. 


\section{Encoding process}

- Compute vector $\left|p_{c}\right\rangle$ as in (8).

- Add $|p\rangle$ and $\left|p_{c}\right\rangle$ to construct

$$
|\tilde{p}\rangle=|p\rangle+\left|p_{c}\right\rangle
$$

\section{Decoding process}

- Use the vector $|\tilde{p}\rangle$ to recover the data $\left|x^{\circ}\right\rangle$ as

$$
\left|x^{o}\right\rangle=\hat{A}|\tilde{p}\rangle
$$

- Use the data $\left|x^{o}\right\rangle$ to determine, by optimisation of $S$, the distribution $|p\rangle$. From $|\tilde{p}\rangle$ and $|p\rangle$ compute vector

$$
\left|p_{c}\right\rangle=|\tilde{p}\rangle-|p\rangle
$$

- Use the decoding operator $\hat{U}$ to obtain the encrypted code by noticing that, since $\hat{U} \hat{U}^{\dagger}$ is the identity operator in $\mathbb{R}^{N-(M+1)}$, from (지) one has:

$$
|q\rangle=\hat{U}\left|p_{c}\right\rangle
$$

Note that the success of the above encoding/decoding scheme relies on the possibility of ordering the eigenvectors in the null space. This is perfectly possible by fixing the numerical method for computing the eigenvectors of $\hat{G}$. However, the process is extremely unstable, as a tiny perturbation to any of the matrix elements of operator $\hat{G}$ produces a huge effect in the eigenvectors of zero eigenvalues. This "chaotic" (in the popular sense) behaviour of the eigenvectors in the null space provides, naturally, the security key for retrieving the encrypted code. Indeed, consider that $\epsilon$ is a very small number (order of $10^{-13}$, say) that we add to one of the matrix elements of $\hat{G}$. Such a tiny perturbation does not yield any detectable effect in the reconstruction 
of the distribution $|p\rangle$ but an enormous effect with regard to the eigenvectors in the null space. Hence, as illustrated by the examples of the next section, the perturbation $\epsilon$ provides the safety key of our encoding/decoding scheme.

\subsection{Numerical Examples}

Consider that a probability distribution concerning an event space of dimension $N=401$ is appropriately determined, by the Jaynes maximum entropy formalism, from the normalisation to unity constraint and the first four moments of a random variable $x_{n}, n=1, \ldots, 401$ that takes values ranging from $x_{1}=-1$ to $x_{401}=1$ with uniform increment $\Delta=1 / 400$. Thus the distribution, which arises by maximisation

of the Shannon's entropy $S=-\sum_{n=1}^{401} p_{n} \ln p_{n}$ subjected to the given constraints, has the form:

$$
\begin{aligned}
p_{n} & =e^{-\lambda_{o}-\lambda_{1} x_{n}-\lambda_{2} x_{n}^{2}-\lambda_{3} x_{n}^{3}-\lambda_{4} x_{n}^{4}} \\
e^{\lambda_{o}} & =\sum_{n=1}^{401} e^{-\lambda_{1} x_{n}-\lambda_{2} x_{n}^{2}-\lambda_{3} x_{n}-\lambda_{4} x_{n}^{4}}
\end{aligned}
$$

The parameters $\lambda_{1}, \lambda_{2}, \lambda_{3}$ and $\lambda_{4}$ are determined from the equations

$$
x_{i}^{o}=\frac{\sum_{n=1}^{401} x_{n}^{i} e^{-\lambda_{1} x_{n}-\lambda_{2} x_{n}^{2}-\lambda_{3} x_{n}^{3}-\lambda_{4} x_{n}^{4}}}{\sum_{n=1}^{401} e^{-\lambda_{1} x_{n}-\lambda_{2} x_{n}^{2}-\lambda_{3} x_{n}^{3}-\lambda_{4} x_{n}^{4}}}, \quad i=1, \ldots, 4
$$

For $x_{1}^{o}=-0.0224, x_{2}^{o}=0.1048, x_{3}^{o}=-0.0124, x_{4}^{o}=0.0284$ one obtains $\lambda_{1}=$ $-0.3, \lambda_{2}=3, \lambda_{3}=2, \lambda_{4}=3.8$. The operator $\hat{A}$ has a $5 \times 401$ matrix representation of elements $x_{i, n}=x_{n}^{i}, i=1, \ldots, 4 ; n=1, \ldots, 401$ and $x_{5, n}=1, n=1, \ldots, 401$. We construct operator $\hat{G}=\hat{A}^{\dagger} \hat{A}$ and compute its eigenvectors. The 396 eigenvectors corresponding to zero eigenvalues are used to construct the encoding operator $\hat{U}^{\dagger}$ to encrypt a code $|q\rangle$ of 396 numbers. These numbers, each of which consists of 15 digits, are taken randomly from the $[0,1]$ interval. We now proceed as indicated in the encoding process of the previous section: We construct the vector $\left|p_{c}\right\rangle=\hat{U}^{\dagger}|q\rangle$ and add it to the distribution $|p\rangle$ to obtain vector $|\tilde{p}\rangle=|p\rangle+\left|p_{c}\right\rangle$. This vector contains both, the information on the physical system and the code. In order to retrieve 
such information we use $|\tilde{p}\rangle$ to generate the constraints as $x_{i}^{o}=\langle i|\hat{A}| \tilde{p}\rangle, i=1, \ldots, 5$. Since, by construction, $\hat{A}\left|p_{c}\right\rangle=0$, the constraints are generated from $|\tilde{p}\rangle$ with high precision. We use then this values to solve for the parameters of the distribution so us to recover $|p\rangle$. Vector $|p\rangle$ allows to obtain vector $\left|p_{c}\right\rangle$ from the available vector $|\tilde{p}\rangle$ as $\left|p_{c}\right\rangle=|\tilde{p}\rangle-|p\rangle$. The code is thus retrieved by the operation $|q\rangle=\hat{U}^{\dagger}\left|p_{c}\right\rangle$. Table 1 gives five of the 396 code numbers. The second column corresponds to the reconstructed numbers. As can be observed the quality of the reconstruction is excellent. In order to give a measure assessing the reconstruction of all numbers, let us denote by $\left|q^{\mathrm{r}}\right\rangle$ the reconstructed code and define the error of the reconstruction as $\left.\delta^{\mathrm{r}}=\||| q\right\rangle-\left|q^{\mathrm{r}}\right\rangle||$. The value of $\delta^{\mathrm{r}}$ is in this case $4.8 \times 10^{-14}$.

Let us now distort the matrix representation of operator $\hat{G}$ by adding a number $\epsilon=2.9 \times 10^{-13}$ to one of its elements, say the element at the first row and fifth column. If we repeat the process using the distorted matrix the outcomes are the following: The perturbation has no detectable effect in the reconstruction of the distribution $|p\rangle$. However, if we intend to reconstruct the code without considering the perturbation, what we obtain has no relation whatsoever with the true code (see the 3rd column of Table 1). The error of the reconstruction is $\delta^{\mathrm{r}}=17.25$. Since the recovery of the code is only possible if the value of the perturbation is known, the key for recovering the code is the value of the perturbation and the to numbers labelling the element that has been distorted, in this case $(1,5)$. Of course, rather than distorting one matrix element we may wish to distort a random number of them. In such a case the decoding key becomes a string of ordered pairs of natural numbers, indicating the elements that were randomly selected to be distorted and the corresponding values of the perturbations. Moreover, to avoid attacks of the type known plaintext attack $[4,5]$, in which the attacker is supposed to have collected correctly decrypted message in order to use them to decrypt others, one can proceed as follows: maintaining one perturbation secret as the key for 


\begin{tabular}{||c|c|c||}
\hline Code numbers & reconstruction & disregarding perturbation \\
\hline 0.43596704982551 & 0.43596704982551 & -0.04525907549619 \\
0.82124392828478 & 0.82124392828478 & 0.18665860768833 \\
0.66471591347310 & 0.66471591347310 & 0.19297513576254 \\
0.64554449242809 & 0.64554449242809 & 0.08172338626606 \\
0.84398334243978 & 0.84398334243978 & -0.14994519073447 \\
\hline
\end{tabular}

Table 1: 5 code numbers and their reconstruction. Assuming the perturbation to be known (second column) and otherwise (third column)

decryption, other perturbations are made public and are different for every message. This avoids the repetition of the encoding operator with the same key. Thus, the knowledge of decrypted messages does not provide information on the encoding operator to encrypt other messages with the same key. This prevents thereby the possibility of known plaintext attacks.

We would like to stress that the success of the proposed procedure strongly depends on the use of the identical numerical method to obtain the same basis of $\operatorname{null}(\hat{G})$ in the encoding and decoding process. Nevertheless, the procedure does not depend on the machine processor. In the examples presented here the encoding was performed in powerful computer cluster, and the decoding in a laptop, using Matlab 6.5. Let us also remark that only the precision in the representation of operator $\hat{G}$ is crucial for the code reconstruction, since the numerical errors in determining $\left|p_{c}\right\rangle$ are not magnified. This is due to the fact that, since $\hat{U}^{\dagger} \hat{U}$ is the identity operator in $\mathbb{R}^{N-(M+1)}$, the inverse recovering of $|q\rangle$ from $|q\rangle=\hat{U}\left|p_{c}\right\rangle$ is very stable against perturbations of $\left|p_{c}\right\rangle$.

\section{Conclusions}

An encoding/decoding scheme for embedding hidden information into the statistic distribution of a physical system has been presented. The encryption security is 
based on the extreme instability of the encoding process, which is endowed with the following feature: a tiny perturbation to the matrix yielding the eigenvalues used to construct the encoding operator produces a huge effect in the recovery process.

Thus, the key for retrieving the code is given by the value of the perturbation. The security for interchanging the key is, of course, essential, but we can rely on the secure quantum key distribution protocol to ensure a safe key delivery. Conversely, the quantum protocol can make use of the proposed setting for encryption, as it entails the transition of very little information through the quantum channel.

A remarkable property of making use of a statistical distribution for the purpose of storing encrypted information is the fact that, the larger the dimension of the distribution is, the larger the amount of encrypted information that can be stored. This opens the possibility of devising more sophisticated encryption algorithms than the one advanced here, yet based on the same principles.

We believe that the results we have presented are certainly encouraging and feel confident that they will contribute to many fruitful discussions and follow-up work in the subject. Finally we would like to stress that the proposed scheme is not restricted to be applied only on physical distributions. When a physical distribution is involved, one has also stored the information on the physical system. Hence the importance of using an appropriate entropic measure for the encoding/decoding process. If the entropic measure is the right one, after recovering the statistical distribution it can be used to make correct predictions on the expected values of physical quantities which are not experimentally available.

\section{References}

[1] Hoi-Kwong Lo, S. Popescu, T Spiller, Eds., Introduction to Quantum Computation and Information, (World Scientific, London, 1998) 
[2] D. Bouwmeester, A. Ekert, A. Zeilinger, Eds., The Physics of Quantum Information, (Springer, London, 2000).

[3] M. A. Nielsen and I. L. Chuang, Quantum Computation and quantum information (Cambridge University Press, UK, 2000)

[4] J. A. Buchmann Introduction to cryptography, (Springer, London, 2004)

[5] B. Schneier Applied Cryptography, (John Wiley \& Sons 1996)

[6] C. H. Bennett, G. Brassard, Proceedings of IEEE International Conference on Computers, Systems and Signal Processing Bangalore, India, (IEEE Press), 175 (1984)

[7] , C. H. Bennett, D. P. DiVicenzo, J. A. Smolin, W. K. Wootters, Phys. Rev. A, 54, 3824 (1996).

[8] P. W. Shor, J. Preskill, Phys. Rev. Lett., 85,2, 441 (2000).

[9] D. Meyers, J. Assoc. Computing Machinery 48,3, 351 (2001)

[10] M. Koashi, J. Preskill, Phys Rev Lett., Feb 7;90(5):057902 (2003)

[11] F. Grosshans, G. Van Assche, J. Wenger, R. Brouri , NJ Cerf, P. Grangier, Nature, Jan 16;421(6920):238-41 (2003)

[12] K. Tamaki, M. Koashi M, N.Imoto Phys Rev Lett., Apr 25;90(16):167904 (2003)

[13] V. Scarani, A. Acin, G. Ribordy, N. Gisin Phys Rev Lett., Feb 6;92(5):057901 (2004)

[14] S. Iblisdir, G. Van Assche, NJ. Cerf Phys Rev Lett., Oct 22;93(17):170502 (2004)

[15] E.T. Jaynes, "Probability theory, the logic of science", (Cambridge University Press, 2003). 
[16] M. S. Baptista. Physics Letters A, (240), 50 (1998).

[17] L. Kocarev, G. Jakimovski, IEEE Trans Circuits and Systems-I, 22 (2001).

[18] J. R. Miotke, L. Rebollo-Neira, Appl. Comput. Harmon. Anal.16,3, 203 (2004). 